\title{
Cultura de segurança do paciente em três hospitais brasileiros com diferentes tipos de gestão
}

\author{
Patient safety culture in three Brazilian hospitals with different \\ types of management
}

Luiz Eduardo Lima Andrade ${ }^{1}$

Johnnatas Mikael Lopes ${ }^{1}$

Marlon César Melo Souza Filho ${ }^{2}$

Romero Fonseca Vieira Júnior ${ }^{2}$

Luiz Paulo Costa Farias ${ }^{2}$

Carla Caroline Medeiros dos Santos ${ }^{2}$

Zenewton André da Silva Gama ${ }^{1}$

${ }^{1}$ Departamento de Saúde Coletiva, Centro de Ciências da Saúde, Universidade Federal do Rio Grande do Norte (UFRN). Av. Sen. Salgado Filho s/n, Lagoa Nova. 59078-970 Natal RN Brasil.

luizeduardofisio@gmail.com

${ }^{2}$ Departamento de

Medicina Integrada, UFRN.

Natal RN Brasil.

\begin{abstract}
The scope of the study was to evaluate patient safety culture and associated factors in Brazilian hospitals with different types of management, namely federal, state and private hospitals. The design was cross-sectional and observational. A survey of 1576 professionals at three hospitals of Rio Grande do Norte state was performed using the Hospital Survey on Patient Safety Culture adapted for Brazil, which measures 12 dimensions of safety culture. Perceptions are described by attributing a general result (Range 0-10) and the percentage of positive responses to estimate their strengths and weaknesses. The response rate was $13.6 \%(n=215)$. The patient safety coefficient was between 7 and 10 for $78.1 \%$ of the respondents, whereby the highest average grade was attributed to the private hospital (8.32). It has been estimated that the type of hospital management, unit service, position and number of adverse event notifications are associated with the overall patient safety grade $(p<0.001)$. Only the private hospital had strengths in the dimensions analyzed, while the weaknesses appeared in all the hospitals.
\end{abstract}

Key words Patient safety, Organizational culture, Safety management, Quality of health care
Resumo O objetivo do estudo foi avaliar a cultura de segurança do paciente e fatores associados em hospitais brasileiros com diferentes tipos de gestão: federal, estadual e privado. O desenho foi observacional transversal. Enviaram-se 1576 questionários aos profissionais de três hospitais do estado do Rio Grande do Norte, utilizando o Hospital Survey on Patient Safety Culture, adaptado para o Brasil, que mede 12 dimensões da cultura de segurança. As percepções são descritas através de uma nota geral ( 0 a 10) e dos percentuais de respostas positivas para estimar fortalezas e fragilidades em cada dimensão. A taxa de resposta foi de13,6\% ( $n=215)$. A segurança do paciente teve nota entre 7 e 10, para $78,1 \%$ dos respondentes, sendo a maior média das notas apresentada pelo hospital privado $(8,32)$. O tipo de gestão hospitalar, unidade de serviço, cargo e quantidade de notificação de eventos adversos estiveram associados à nota geral da segurança do paciente $(p<0,001)$. Apenas o hospital privado apresentou fortalezas nas dimensões analisadas, enquanto que as fragilidades apareceram em todos os hospitais.

Palavras-chave Segurança do paciente, Cultura organizacional, Gerenciamento de segurança, Qualidade da assistência à saúde 


\section{Introdução}

Incidentes que afetam a Segurança do Paciente (SP) ocorrem o ano inteiro em hospitais em todo o mundo. Nos Estados Unidos, estimou-se que entre 44 mil e 98 mil pessoas morrem por ano devido a erros e EA evitáveis ${ }^{1}$, um problema maior que os acidentes de trânsito, câncer de pulmão ou AIDS. No Brasil, a situação não é menos grave, uma vez que o país possui uma das maiores frequências de Eventos Adversos (EA) evitáveis do mundo ${ }^{2}$.

A SP consiste na redução, a um mínimo aceitável, do risco de dano desnecessário associado ao cuidado de saúde ${ }^{3}$. Ela é uma dimensão da qualidade em cuidados de saúde $e^{4}$ e melhorá-la depende de aprender a lidar com as interações dos seus componentes. O Instituto de Medicina dos Estados Unidos recomendou que as organizações de saúde criassem um ambiente onde a SP fosse um objetivo organizacional explícito e ocupasse o topo das prioridades das lideranças ${ }^{5}$, tornando-se parte importante da cultura organizacional.

A Cultura de Segurança do Paciente (CSP) é considerada um importante componente estrutural dos serviços que favorece a implantação de práticas seguras e diminuição de incidentes de segurança. O termo cultura de segurança foi utilizado inicialmente pela International Atomic Energy Agency (IAEA), quando publicou o Safety Culture: a Report by International Nuclear Safety Advisory Group (INSAG-4) ${ }^{6}$. No âmbito da saúde, a CSP foi definida pela Agency for Healthcare Research and Quality (AHRQ) como o produto de valores, atitudes, percepções, competências e padrões de comportamento de grupos e de indivíduos. Isto determina o compromisso, estilo e proficiência no manejo de segurança em saúde de uma organização ${ }^{7}$.

Avaliar a cultura de segurança é importante para medir as condições organizacionais que levam a possíveis danos ao paciente nos serviços de saúde ${ }^{8}$. Esse tipo de avaliação tem várias utilidades: diagnosticar o nível de cultura de segurança, possíveis riscos de dano, benchmarking interno e externo, evolução das intervenções de SP e acompanhar evolução da CSP com o tempo, bem como a notificação de incidentes. Avaliar a cultura de segurança depende do envolvimento das partes interessadas. Deve-se selecionar uma ferramenta adequada, utilizar métodos de coletas de dados válidos, implementar o plano de ação e iniciar as mudanças?
Várias são as ferramentas para mensurar a CSP, como listas de verificação, avaliação de risco ambiental, entrevistas estruturadas, análise de causa-efeito ${ }^{10}$, sendo a avaliação via questionário de autopreenchimento o método mais utilizado devido à rapidez de ser distribuído por grandes grupos em pouco tempo, além de ser aplicado facilmente. Entre eles o Hospital Survey on Patient Safety Culture (HSOPSC) é um dos que possuem capacidade de fornecer aos profissionais, gestores e dados mais precisos e extensos acerca da CSP de um serviço de saúde, podendo servir de comparativo contra outras unidades de saúde ou hospitais ${ }^{11}$.

No Brasil, pesquisas foram desenvolvidas com objetivo de analisar a cultura de segurança do paciente na perspectiva de unidades hospitalares, ou por categorias profissionais específicas, utilizando o HSOPSC ${ }^{12,13}$, ou o Questionário de Atitudes de Segurança ${ }^{14,15}$. Nestes estudos foram analisadas as principais fragilidades e fortalezas das dimensões que formam o constructo "Cultura de Segurança" nos instrumentos, servindo de base para a construção de medidas de intervenção em dimensões consideradas frágeis.

Para fomentar a implementação de iniciativas voltadas à segurança do paciente, foi instituído o Programa Nacional de Segurança do Paciente (PNSP), em abril de $2013^{16}$. A Resolução da Diretoria Colegiada (RDC), da Agência Nacional de Vigilância Sanitária (Anvisa), no 36 de 2013, regulamenta as ações para a SP em serviços de saúde, criando os Núcleos de Segurança do Paciente (NSP), que apresentam como uma de suas funções a promoção da CSP mediante a implementação de Planos de Segurança do Paciente nos estabelecimentos de saúde ${ }^{17}$.

Neste contexto político de incentivo à cultura de segurança, ainda não há informações sobre os diferentes níveis de CSP em hospitais brasileiros, estabelecimentos que possuem características diversas e diferentes arranjos organizacionais. Avaliar a CSP e seus determinantes em hospitais com diferentes tipos de gestão sinalizam para possíveis intervenções de melhoria necessárias para impulsionar a qualidade da assistência e segurança do paciente neste contexto. Medir a CSP também é uma atividade básica para promovê $-\mathrm{la}^{18}$, pois constrói uma linha de base e possibilita o monitoramento dos efeitos das intervenções internas e das políticas públicas. Portanto, o objetivo deste estudo é avaliar a cultura de segurança do paciente e fatores associados em hospitais brasileiros com diferentes tipos de gestão. 


\section{Método}

\section{Desenho do estudo}

Trata-se de um delineamento observacional do tipo transversal, com abordagem analítica. A coleta de dados foi realizada em três hospitais do estado do Rio Grande do Norte, Brasil, com diferentes tipos de gestão, a saber: federal, estadual e privada. O projeto foi desenvolvido no período de outubro (coleta via email) a novembro (coleta presencial) de 2013, seis meses após a instituição do PNSP e um mês após a publicação da RDC $36 / 2013$.

\section{Participantes}

A seleção dos hospitais participantes foi por conveniência e tentando assegurar o de maior porte no município do estudo em cada categoria (federal, estadual e privado).

O hospital estadual selecionado é o de maior porte entre os 24 hospitais públicos estaduais do RN. É um hospital geral com pronto atendimento, sendo referência em Traumatologia e Urgência para o estado, apresentando 270 leitos. $\mathrm{O}$ arranjo organizacional é de administração direta pelo governo estadual. O Núcleo de Segurança do Paciente estava em fase de constituição e o serviço não possuia sistema de notificação interno de incidentes. O serviço possuia Núcleo de Educação Permanente (NEP) implantado.

O hospital privado selecionado é um dos três maiores dentre os hospitais privados do estado, e ainda é o único hospital do RN com selo de acreditação (nível 1) da Organização Nacional de Acreditação (ONA). É um hospital geral com pronto atendimento e possui 86 leitos. Seu arranjo organizacional é privado sem fins lucrativos, sendo administrado por uma cooperativa médica. Até o período da coleta não teve implementado o NSP nem o sistema de notificação de EA interno, mas possuía NEP. Ele não faz parte da rede sentinela e tem como público alvo cidadãos de classe média e alta. O hospital público federal selecionado é o maior dentre os quatro hospitais federais do estado. Seu arranjo organizacional é de administração indireta por meio de uma autarquia, que é a Universidade Federal do Rio Grande do Norte. O hospital é geral, sem pronto atendimento, e possui 240 leitos. Ele tinha Núcleo de Segurança do Paciente (NSP) recém implantado, sistema de notificação de incidentes interno e núcleo de educação permanente. Tratase de um hospital universitário, certificado como hospital de ensino e pertencente à rede de hospitais sentinela.

Quanto aos profissionais, participaram da pesquisa os que possuíam algum vínculo contratual, fixo ou temporário ligado ao centro hospitalar, sejam aqueles vinculados à assistência (médicos, enfermeiros, farmacêuticos e outros), bem como os relacionados à gestão. Além disso, a fim de tornar fidedignas as informações dos hospitais e diminuir os riscos de incluir na amostra os profissionais que não trabalham na instituição, ao se encaminhar os questionários, foram descartados os profissionais que estavam fazendo substituições e que não pertenciam ao quadro.

\section{Instrumento de coleta}

$\mathrm{O}$ instrumento utilizado para a coleta foi o questionário HSOPSC adaptado à realidade brasileira $^{19}$, acrescido de algumas oportunidades de melhoria $^{20}$. Ele contém 42 questões, medindo 12 Dimensões (D) da cultura de segurança:

D1. Frequência de eventos notificados (3 itens);

D2. Percepção de segurança (4 itens);

D3. Expectativas e ações da direção/supervisão da unidade/serviços que favoreçam a segurança (4 itens);

D4. Aprendizagem organizacional / melhoria continuada (3 itens);

D5. Trabalho em equipe na unidade / serviço (4 itens);

D6. Abertura para comunicações (3 itens);

D7. Feedback e comunicação sobre erros (3 itens);

D8. Resposta não punitiva para erros (3 itens);

D9. Dimensionamento de pessoal (4 itens);

D10. Apoio da gerência do hospital para a segurança do paciente (3 itens);

D11. Trabalho em equipe entre unidades (4 itens);

D12. Problemas em mudanças de turno e transições entre unidades/serviços (4 itens).

As 42 questões relativas à CSP foram construídas baseadas em uma escala tipo likert de 5 alternativas: discordo totalmente; discordo; nem concordo nem discordo; concordo; concordo totalmente; ou nunca; quase nunca; às vezes; quase sempre; e sempre. O questionário ainda contém uma pergunta de qualificação global do nível de segurança do paciente (0-10) e uma questão sobre o número de incidentes de segurança notificados no último ano.

O instrumento possui itens construídos positivamente, ou seja, a resposta concordante a estes 
é positiva para a cultura de segurança, bem como itens escritos negativamente, cujas respostas discordantes são igualmente positivas para cultura de segurança.

\section{Coleta de dados}

Inicialmente, foi repassada, pelos setores responsáveis de cada hospital, uma lista contendo o endereço eletrônico dos profissionais cadastrados para envio do questionário, por e-mail. Com intuito de se obter o máximo de respostas possíveis, foram feitas três tentativas via e-mail para cada profissional, no prazo de 7, 10 e 15 dias após a primeira submissão do questionário. Posteriormente, para ampliar o percentual de respostas, a coleta de dados foi complementada presencialmente com o preenchimento dos questionários por meio de dispositivos eletrônicos: tablets ou smartphones, através do software ad hoc, desenvolvido para coleta e análise automática de dados: E-Questionário de Cultura de Segurança do Paciente Hospitalar. Neste caso, a coleta presencial foi realizada em diferentes turnos e participaram os profissionais que não responderam o questionário via correio eletrônico, agregando também os que não possuíam e-mail.

\section{Variáveis de interesse}

As variáveis relacionadas ao nível de cultura de segurança do paciente foram a nota global de segurança fornecida pelos respondentes, variando de 0 a 10 ( 1 e 2, péssimo; 3 e 4, ruim; 5 e 6, regular; 7 e 8, bom; e 9 e 10, excelente), e a porcentagem de respostas positivas às perguntas relacionadas a cada dimensão da cultura de segurança do paciente.

A fim de se estimar a influência do tipo de gestão e as características dos servidores foram conceituadas como variáveis potencialmente preditoras do nível de cultura de segurança do paciente os anos de trabalho na profissão, anos de trabalho na unidade, horas de trabalho semanais, profissão, cargo, unidade ou serviço que atua e tipo de gestão hospitalar (público federal, público estadual ou privado).

\section{Análise dos dados}

A classificação do grau de percepção de segurança é descrita usando a mediana e os percentis 25 e 75 da questão sobre nota global da segurança do paciente. Além disso, foram descritas as porcentagens de respostas positivas em cada uma das dimensões da cultura de segurança e no total de dimensões, acompanhados de seus respectivos intervalos de confiança de 95\% (IC95\%), que servem para classificar as dimensões em fortalezas quando os itens apresentam $75 \%$ ou mais de respostas positivas (respondendo concordo ou concordo totalmente aos itens escritos positivamente ou discordo ou discordo totalmente aos itens escritos negativamente) e fragilidade cujos percentuais de repostas positivas são iguais ou inferiores a $50 \%$. Foi ainda descrita a média da nota geral para a segurança do paciente por hospital e no agregado destes.

Para verificar as possíveis interveniências dos fatores organizacionais sobre a nota geral para a segurança do paciente, foi utilizado a Generalized Estimated Equation (GEE), sendo levado em conta os seguintes parâmetros: Intercepto que representa o ponto no modelo onde os valores das variáveis independentes equivalem a zero, e B ou Coeficiente de inclinação da variável, nota geral de segurança do paciente em função da variável independente. Foram também utilizadas as seguintes estratégias de modelagem: matriz de correlação não estruturada, função ligante de Poisson e teste de hipótese de $\mathrm{x}^{2}$ de Wald. Foi adotado um nível de significância de 5\% para minimizar um erro tipo I.

O presente estudo contou com financiamento interno do projeto de ações integradas da Universidade Federal do Rio Grande do Norte (UFRN) e cumpriu os preceitos éticos para pesquisa, com projeto submetido e aprovado no Comitê de Ética em Pesquisa da UFRN.

\section{Resultados}

\section{Caracterização da amostra}

As listas enviadas pelos três hospitais totalizaram 1.576 profissionais, que receberam em seus e-mails o questionário para o preenchimento. Dos questionários enviados 215 (13,6\%) foram respondidos, sendo a porcentagem de respostas semelhante nos três hospitais, com $72(18,6 \%)$ no hospital federal, $84(10,6 \%)$ no estadual e 59 $(15,6 \%)$ no privado. Entre os profissionais que responderam a pesquisa, $102(48,2 \%)$ eram da equipe de enfermagem, 23 (10,6\%) médicos, 14 $(6,9 \%)$ eram fisioterapeutas terapeutas ocupacionais ou fonoaudiólogos e 6 (2,3\%) farmacêuticos. Entre os respondentes, 161 (78,5\%) têm contato direto com os pacientes em seu trabalho, $106(56,6 \%)$ trabalham mais de 40 horas sema- 
nais e apenas um profissional trabalha há menos de um ano na instituição (Tabela 1).

De uma maneira geral, 55,8\% dos profissionais dos três hospitais classificaram a SP como boa (nota 7 ou 8 ) ou excelente (nota 9 ou 10) e $22,3 \%$ classificaram como péssima (nota 1 ou 2 ) ou ruim (nota 3 ou 4 ).

Em todos os hospitais foi encontrada uma média de $38 \%$ para a proporção de respostas positivas, esta média reflete uma percepção geral de todas as dimensões da cultura de segurança (Tabela 2). Ao se analisar todos os hospitais, a frequência de respostas positivas para as dimensões revelou percentuais de resposta mais altos nas dimensões "expectativas e ações da direção/ supervisão da unidade/serviço que favorecem a segurança" e "trabalho em equipe na unidade/ serviço" com 66,7\% e 60,8\%, respectivamente. No entanto, de acordo com a AHRQ, nenhuma dimensão atingiu o nível de fortaleza da cultura de segurança no agregado de hospitais, já que isso só ocorre com mais de 75\% de respostas positivas para uma dada dimensão.

Ainda sobre o total de hospitais, analisou-se que sete dimensões foram frágeis (porcentagem de resposta positiva menor que 50\%): "Frequência de eventos notificados", "Percepção de segurança", "Feedback e comunicação sobre os erros", "Resposta não punitiva para erros", "Dimensionamento de pessoal", "Trabalho em equipe entre unidades" e "Problemas em mudanças de turno e transições entre unidades/serviços” (Tabela 3).

\section{Comparação do nível de segurança em hospitais com diferentes tipos de gestão}

Quanto à nota geral para a segurança do paciente, o hospital privado foi o que apresentou melhor média das três instituições $(8,32)$ (Tabela 2). Mesmo assim, 62,9\% dos participantes do hospital federal classificaram a nota como boa (nota 7 ou 8 ) ou excelente (nota 9 ou 10) e 25,9\% dos respondentes no hospital estadual. Neste último, 48,2\% dos profissionais avaliaram como péssima (nota 1 ou 2) ou ruim (nota 3 ou 4), enquanto, no federal, apenas $10 \%$ fizeram a mesma classificação e, no privado, nenhum profissional classificou desta forma.

Ao se considerar separadamente as dimensões avaliadas em cada hospital, percebe-se que apenas o hospital privado conseguiu atingir o nível de fortaleza definido pela AHRQ nas dimensões "expectativas e ações da direção/supervisão da unidade/serviço que favorecem a segurança", com 86,4\%; "aprendizagem organizacional/me- lhoria continuada", com 87,6\%; "Trabalho em equipe na unidade/serviço", com 76,8\%; "Apoio da gerência do hospital para a segurança do paciente", com 90,2\%; e "Trabalho em equipe entre unidades”, com 77,3\% (Tabela 3).

No tocante às dimensões avaliadas com baixo percentual de respostas positivas $(<50 \%)$, todos os hospitais apresentaram dimensões que atingem níveis de fragilidade, de acordo com a AHRQ. Sendo o estadual o único a apresentar as 12 dimensões com fragilidades, seguido do federal com 6 dimensões e o privado com apenas uma dimensão classificada como fragilidade (Tabela 3 ).

Ao se comparar as porcentagens de respostas positivas de cada hospital (Figura 1), em todas as dimensões, é visto que o federal apresenta valores intermediários entre as porcentagens obtidas pelos demais hospitais, sendo o privado o que apresentou melhores porcentagens de respostas positivas em todas as dimensões, e o hospital estadual o que obteve as menores porcentagens positivas em relação aos três tipos de estabelecimentos avaliados.

O modelo de predição para a nota geral da segurança fornecida por cada um dos respondentes, de acordo com a modelagem utilizada, foi evidenciada a distinção entre os hospitais quanto à nota geral (Wald x2 = 246,37; p < 0,001), onde os funcionários do hospital federal $(B=0,35 ; \mathrm{p}<0,01)$ e do privado $(B=0,55 ; p<0,01)$, em relação ao estadual, relatam maiores notas. Outra característica que demostra interferência na nota é a unidade de trabalho do respondente (Wald x2 = 73,00; $\mathrm{p}<$ $0,001)$, onde as unidades de emergência, psiquiatria e radiologia tendem a relatar menores notas sobre a segurança do paciente, enquanto que a farmácia dá notas maiores à SP do serviço quando comparados às outras unidades (Tabela 4).

Foi revelado também que o cargo ocupado pelos funcionários é interveniente na estimativa da nota geral SP (Wald $\mathrm{x} 2=512,31 ; \mathrm{p}<0,001$ ). Parece que os cargos de médico do corpo clínico, farmacêutico e assistente social têm a tendência de fornecer notas mais baixas que o cargo de odontólogo, enquanto que médicos residentes fornecem maiores escores, assim como fisioterapeutas, fonoaudiólogos e terapeuta ocupacional, em relação a outras profissões. Outro fator interveniente é a quantidade de notificações de incidentes, isto é, aqueles profissionais que fazem mais notificações fornecem as maiores notas gerais da segurança do paciente. Não foi encontrado qualquer valor de predição para as variáveis tempo de trabalho, tempo na área e horas trabalhada semanais $(\mathrm{p}>0,05)$ (Tabela 4$)$. 
Tabela 1. Caracterização dos profissionais respondentes nos três hospitais participantes, Rio Grande do Norte, Brasil, 2013.

\begin{tabular}{|c|c|c|c|c|}
\hline Variáveis & $\begin{array}{c}\text { Hospital Federal } \\
\mathbf{n}=72 \\
\mathbf{n}(\%)\end{array}$ & $\begin{array}{c}\text { Hospital Estadual } \\
\mathbf{n}=\mathbf{8 4} \\
\mathbf{n}(\%)\end{array}$ & $\begin{array}{c}\text { Hospital Privado } \\
\text { n=59 } \\
\mathbf{n}(\%)\end{array}$ & $\begin{array}{c}\text { Total } \\
\mathbf{n}=215 \\
\mathrm{~N}(\%) \\
\end{array}$ \\
\hline \multicolumn{5}{|l|}{ Contato com o paciente } \\
\hline $\operatorname{Sim}$ & $58(80,5)$ & $62(73,8)$ & $41(75,9)$ & $161(75,8)$ \\
\hline Não & $11(15,2)$ & $20(23,8)$ & $13(22,0)$ & $44(20,4)$ \\
\hline Missing & $3(4,30)$ & $2(2,40)$ & $5(2,1)$ & $10(3,8)$ \\
\hline \multicolumn{5}{|l|}{$\begin{array}{l}\text { Tempo de trabalho na especialidade/ } \\
\text { profissão atual }\end{array}$} \\
\hline Menos que 1 ano & $0(0)$ & $0(0)$ & $1(1,6)$ & $1(0,46)$ \\
\hline De 1 a 5 anos & $25(34,7)$ & $15(17,9)$ & $16(27,1)$ & $56(26,0)$ \\
\hline De 6 a 10 anos & $10(13,8)$ & $22(26,2)$ & $16(27,1)$ & $48(22,3)$ \\
\hline De 11 a 15 anos & $11(15,2)$ & $4(4,70)$ & $13(22,0)$ & $28(13,0)$ \\
\hline De 16 a 20 anos & $10(13,8)$ & $7(8,30)$ & $4(5,55)$ & $21(9,76)$ \\
\hline De 21 anos ou mais & $10(13,8)$ & $36(42,9)$ & $3(5,08)$ & $49(22,7)$ \\
\hline Missing & $16(22,2)$ & $0(0)$ & $6(11,5)$ & $12(5,78)$ \\
\hline \multicolumn{5}{|l|}{ Tempo de trabalho neste hospital } \\
\hline Menos que 1 ano & $0(0)$ & $0(0)$ & $1(1,90)$ & $1(0,50)$ \\
\hline De 1 a 5 anos & $39(58,2)$ & $34(40,5)$ & $31(57,4)$ & $104(50,7)$ \\
\hline De 6 a 10 anos & $7(10,4)$ & $4(4,80)$ & $19(35,2)$ & $30(14,6)$ \\
\hline De 11 a 15 anos & $3(4,50)$ & $12(14,3)$ & $1(1,90)$ & $16(7,80)$ \\
\hline De 16 a 20 anos & $9(13,4)$ & $12(14,3)$ & $0(0)$ & $21(10,2)$ \\
\hline De 21 anos ou mais & $5(7,50)$ & $3(3,60)$ & $2(3,70)$ & $10(4,90)$ \\
\hline \multicolumn{5}{|l|}{$\begin{array}{l}\text { Tempo de trabalho na área/unidade } \\
\text { atual }\end{array}$} \\
\hline Menos que 1 ano & $0(0)$ & $1(1,20)$ & $3(5,50)$ & $4(2,00)$ \\
\hline De 1 a 5 anos & $41(62,1)$ & $47(56,6)$ & $37(67,3)$ & $125(61,3)$ \\
\hline De 6 a 10 anos & $8(12,1)$ & $4(4,80)$ & $14(25,5)$ & $26(12,8)$ \\
\hline De 11 a 15 anos & $7(10,6)$ & $7(8,40)$ & $0(0)$ & $14(6,90)$ \\
\hline De 16 a 20 anos & $3(4,50)$ & $10(12,0)$ & $0(0)$ & $13(6,40)$ \\
\hline De 21 anos ou mais & $5(7,60)$ & $5(6,00)$ & $1(1,80)$ & $11(5,40)$ \\
\hline \multicolumn{5}{|l|}{ Carga horária semanal } \\
\hline Menos que 20 horas & $8(11,6)$ & $5(6,20)$ & $6(11,3)$ & $19(9,70)$ \\
\hline Entre 20 e 39 horas & $31(44,9)$ & $25(30.9)$ & $22(41,5)$ & $73(38,4)$ \\
\hline Entre 40 e 59 horas & $28(40,6)$ & $50(61,7)$ & $25(47,2)$ & $103(50,7)$ \\
\hline Mais de 60 horas & $2(2,80)$ & $1(1,20)$ & $0(0)$ & $3(1,50)$ \\
\hline \multicolumn{5}{|l|}{ Profissão } \\
\hline Médicos & $7(9,72)$ & $14(16,6)$ & $2(3,38)$ & $23(10,6)$ \\
\hline Enfermeiros & $16(22,2)$ & $15(17,8)$ & $7(11,8)$ & $38(17,6)$ \\
\hline Técnicos de enfermagem & $21(29,1)$ & $15(17,8)$ & $28(47,4)$ & $64(29,7)$ \\
\hline Farmacêuticos & $1(1,38)$ & $2(2,38)$ & $3(5,08)$ & $6(2,79)$ \\
\hline $\begin{array}{l}\text { Fisioterapeutas, terapeutas } \\
\text { ocupacionais e fonoaudiólogos }\end{array}$ & $4(5,55)$ & $10(11,9)$ & $0(0)$ & $14(6,51)$ \\
\hline Missing & $23(31,9)$ & $28(33,3)$ & $19(32,2)$ & $70(32,5)$ \\
\hline \multicolumn{5}{|l|}{ Serviço } \\
\hline Medicina clínica & $22(28,9)$ & $12(14,3)$ & $14(24,1)$ & $48(22,0)$ \\
\hline Cirurgia & $13(17,1)$ & $4(4,80)$ & $10(17,2)$ & $27(12,4)$ \\
\hline Farmácia & $1(1,30)$ & $2(2,40)$ & $4(6,90)$ & $7(3,20)$ \\
\hline Emergência & $0(0)$ & $12(14,3)$ & $3(5,20)$ & $15(6,90)$ \\
\hline UTI & $9(11,8)$ & $10(11,9)$ & $8(13,8)$ & $27(12,4)$ \\
\hline Outros & $31(40,8)$ & $44(52,3)$ & $19(32,8)$ & $94(43,1)$ \\
\hline
\end{tabular}


Tabela 2. Porcentagem de respostas positivas e média da nota geral para a segurança do paciente por hospital e total, Brasil, 2013.

\begin{tabular}{lcccc}
\hline \multirow{1}{*}{ Indicadores } & Hospital Estadual & Hospital Federal & Hospital Privado & \multicolumn{2}{c}{ Total } \\
& $\mathbf{n = 8 4}$ & $\mathbf{n}=\mathbf{7 2}$ & $\mathbf{n}=\mathbf{5 9}$ & $\begin{array}{c}\mathbf{n}=\mathbf{2 1 5} \\
\text { \% }\end{array}$ \\
\hline PRP & $26,5(23,4-29,5)$ & $38,6(35-42,2)$ & $53,8(50,3-57,4)$ & $38(35,6-40,5)$ \\
Nota & $4,68(4,19-5,17)$ & $6,73(6,35-7,12)$ & $8,32(8-8,64)$ & $6,36(6,04-6,68)$ \\
\hline
\end{tabular}

$\mathrm{PRP}=$ Porcentagem de Respostas Positivas. NOTA = Média das notas gerais a segurança do paciente. OBS: A PRP não totaliza os $100 \%$ devido a possibilidade de respostas neutras e negativas.

Tabela 3. Percentual de respostas positivas segundo as dimensões do questionário, Brasil, 2013.

\begin{tabular}{|c|c|c|c|c|c|c|c|c|}
\hline \multirow{3}{*}{ Dimensões } & \multirow{2}{*}{\multicolumn{2}{|c|}{$\begin{array}{c}\text { Hospital Estadual } \\
\text { Positivas }\end{array}$}} & \multirow{2}{*}{\multicolumn{2}{|c|}{$\begin{array}{c}\text { Hospital Federal } \\
\text { Positivas }\end{array}$}} & \multirow{2}{*}{\multicolumn{2}{|c|}{$\begin{array}{c}\text { Hospital Privado } \\
\text { Positivas }\end{array}$}} & \multirow{2}{*}{\multicolumn{2}{|c|}{$\begin{array}{c}\text { Total } \\
\text { Positivas }\end{array}$}} \\
\hline & & & & & & & & \\
\hline & $\%$ & IC95\% & $\%$ & IC95\% & $\%$ & IC95\% & $\%$ & IC95\% \\
\hline $\begin{array}{l}1 \text { - frequência de eventos } \\
\text { notificados }\end{array}$ & $30,8^{*}$ & $20,9-40,6$ & $31,4^{*}$ & $20,9-41,8$ & 61,5 & $48,9-74$ & $39,2^{*}$ & $32,7-45,6$ \\
\hline 2 - percepção de segurança & $21,6^{*}$ & $12,7-30,4$ & $37,5^{*}$ & $26,6-48,3$ & 63,2 & $50,7-75,6$ & $38,2^{*}$ & $31,7-44,6$ \\
\hline $\begin{array}{l}3 \text { - expectativas e ações da } \\
\text { direção/supervisão da unidade/ } \\
\text { serviço que favorecem a } \\
\text { segurança }\end{array}$ & $50,0^{*}$ & $39,3-60,6$ & 70,0 & $59,6-80,3$ & $86,4^{+}$ & $77,5-95,2$ & 66,7 & $60,4-72,9$ \\
\hline $\begin{array}{l}4 \text { - aprendizagem } \\
\text { organizacional/melhoria } \\
\text { continuada }\end{array}$ & $45,9^{*}$ & $35,2-56,5$ & 54,5 & $43,3-65,6$ & 87,6 & $6+79,1-96$ & 60,0 & $53,4-66,5$ \\
\hline $\begin{array}{l}5 \text { - trabalho em equipe na } \\
\text { unidade/serviço }\end{array}$ & $46,2^{\star}$ & $35,5-56,8$ & 64,8 & $54-75,5$ & $76,8^{+}$ & $65,9-87,6$ & 60,8 & $54,3-67,2$ \\
\hline 6 - abertura para comunicações & $43,2^{\star}$ & $32,6-53,7$ & 58,1 & $47-69,1$ & 72,5 & $61,0-83,9$ & 56,2 & $49,6-62,7$ \\
\hline $\begin{array}{l}7 \text { - feed-back e comunicação } \\
\text { sobre erros }\end{array}$ & $28,3^{*}$ & $18,6-37,9$ & $35,8^{*}$ & + $25-46,5$ & $70,4($ & $(58,6-82,1)$ & $42,1^{*}$ & $35,5-48,6$ \\
\hline $\begin{array}{l}8 \text { - resposta não punitivas para } \\
\text { erros }\end{array}$ & $33,3^{*}$ & $23,2-43,3$ & $31,3^{*}$ & $20,8-41,7$ & $46,3^{*}$ & $33,4-59,1$ & $36,1^{\star}$ & $29,6-42,4$ \\
\hline $\begin{array}{l}9 \text { - dimensionamento de } \\
\text { pessoal }\end{array}$ & $30,1^{\star}$ & $20,2-39,9$ & $43,0^{*}$ & $31,8-54,1$ & 70,1 & $58,3-81,8$ & $45,2^{\star}$ & $38,5-51,8$ \\
\hline $\begin{array}{l}10 \text { - apoio da gerência do } \\
\text { hospital para a segurança do } \\
\text { paciente }\end{array}$ & $26,1^{\star}$ & $16,7-35,4$ & 51,6 & $40,3-62,8$ & $90,2^{+}$ & $82,5-97,8$ & 52,1 & $45,4-58,7$ \\
\hline $\begin{array}{l}11 \text { - trabalho em equipe entre } \\
\text { unidades }\end{array}$ & $22,9^{*}$ & $13,9-31,8$ & 52,9 & $41,6-64,1$ & $77,3^{+}$ & $66,5-88,0$ & $47,8^{\star}$ & $41,1-54,4$ \\
\hline $\begin{array}{l}12 \text { - problemas em mudanças } \\
\text { de turno e transições entre } \\
\text { unidades/serviços }\end{array}$ & $19,7^{\star}$ & $11,1-28,2$ & $40,7^{\star}$ & $36,3-58,8$ & 60,0 & $47,3-72,6$ & $37,7^{\star}$ & $31,2-44,1$ \\
\hline
\end{tabular}

* Dimensões classificadas como fragilidades; ${ }^{\dagger}$ Dimensões classificadas como fortalezas.

\section{Discussão}

Este estudo contribui originalmente para a compreensão sobre os níveis de CSP no contexto dos hospitais brasileiros e o conhecimento de fatores que influenciam este aspecto da psicologia das organizações de saúde. No Brasil, ainda não foram divulgadas pesquisas que contemplem a análise de fatores associados à CSP em hospitais, e sua comparação em relação aos diferentes tipos de gestão dos mesmos. As poucas fortalezas identificadas revelam uma incipiente CSP nos hospitais pesquisados, apesar dos respondentes referirem, em sua maioria, que a SP é boa. Percebe-se ainda que o tipo de gestão, unidade do serviço, o cargo e a quantidade de notificações de incidentes influenciam na nota geral da SP.

A SP foi classificada como boa ou excelente pela maioria $(55,8 \%)$ dos respondentes. Esta percepção para as instituições avaliadas é ligei- 

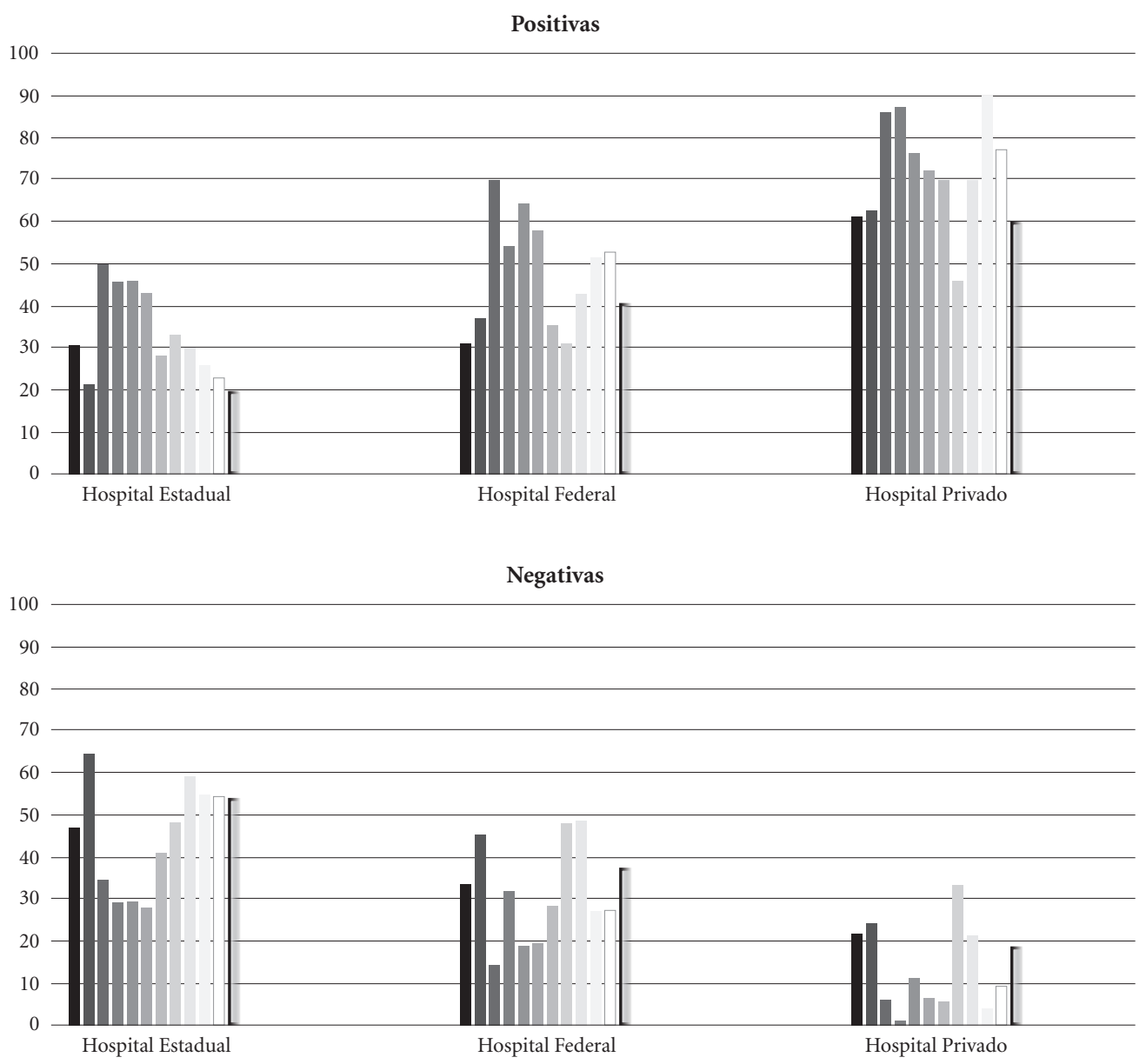

$\square \mathrm{D} 1 \square \mathrm{D} 2 \square \mathrm{D} 3 \quad \square \mathrm{D} 4 \square \mathrm{D} 5 \square \mathrm{D} 6 \square \mathrm{D} 7 \quad \mathrm{D} 8 \quad \mathrm{D} 9 \quad \mathrm{D} 10 \square \mathrm{D} 11 \quad[\mathrm{D} 12$

Figura 1. Diagrama do percentual de respostas positivas e negativas às dimensões do questionário, por hospital. Brasil, 2015.

ramente maior que as encontradas em estudos prévios realizados em outras nacionalidades, os quais avaliaram a cultura de segurança utilizando a versão original do questionário e identificaram que menos de 50\% dos participantes atribuíram uma pontuação maior ou igual a 7,021,22 .

As áreas prioritárias para ações que visem melhoria da cultura de segurança nos hospitais estudados devem focar as dimensões " Frequência de eventos notificados", "Percepção de segurança", "Feedback e comunicação sobre os erros", "Resposta não punitiva para erros", "Dimensionamento de pessoal", "Trabalho em equipe entre unidades" e "Problemas em mudanças de turno e transições entre unidades/serviços”, pois são as áreas em que ocorrem os menores percentuais de respostas positivas quando se analisa o agregado de hospitais. Ao mesmo tempo, deve-se aproveitar e reforçar as melhores condições encontradas nas dimensões da cultura de segurança "expectativas e ações da direção/supervisão da unidade/ serviço que favorecem a segurança”, "aprendizagem organizacional/melhoria continuada" e "trabalho em equipe na unidade/serviço".

A fragilidade no dimensionamento de pessoal pode ser reflexo da insatisfação com as condições de trabalho, a carga excessiva de trabalho, jornada de trabalho desgastante e trabalho sob pressão. Alguns estudos demonstram a relação dessa dimensão com os índices de segurança do paciente ${ }^{23,24}$. Quanto à resposta punitiva por erros cometidos, pode ocasionar limitações no 
Tabela 4. Modelo de predição para a nota geral da segurança do paciente em três serviços hospitalares do Rio Grande do Norte, Brasil, 2013.

\begin{tabular}{|c|c|c|c|c|c|c|c|}
\hline \multirow{2}{*}{ Variáveis } & \multirow{2}{*}{$\%$} & \multirow{2}{*}{ B } & \multicolumn{2}{|c|}{ (IC95\%) } & \multicolumn{3}{|c|}{ Teste de Hipótese } \\
\hline & & & Inferior & Superior & Wald $x^{2}$ & gl & Sig. \\
\hline Intercepto & & 1,63 & 1,34 & 1,91 & 124,44 & 1 & $<0,001$ \\
\hline \multicolumn{8}{|l|}{ Hospitais } \\
\hline Federal & 33,4 & 0,35 & 0,22 & 0,49 & 25,52 & 1 & $<0,001$ \\
\hline Privado & 27,6 & 0,55 & 0,42 & 0,69 & 65,72 & 1 & $<0,001$ \\
\hline Estadual & 39 & 0 & & & & & \\
\hline \multicolumn{8}{|l|}{ Unidade } \\
\hline Diversas unidades & 15,9 & $-0,03$ & $-0,18$ & 0,11 & 0,21 & 1 & 0,64 \\
\hline Medicina Clínica & 15 & $-0,14$ & $-0,30$ & 0,01 & 3,52 & 1 & 0,06 \\
\hline Cirurgia & 12,1 & $-0,03$ & $-0,18$ & 0,10 & 0,24 & 1 & 0,62 \\
\hline Obstetrícia & 1,9 & $-0,01$ & $-0,62$ & 0,61 & 0,01 & 1 & 0,99 \\
\hline Emergência & 7 & $-0,46$ & $-0,76$ & $-0,160$ & 9,00 & 1 & $<0,01$ \\
\hline UTI & 12,6 & $-0,04$ & $-0,18$ & 0,09 & 0,38 & 1 & 0,53 \\
\hline Psiquiatria & 0,9 & $-0,30$ & $-0,48$ & $-0,12$ & 11,26 & 1 & 0,001 \\
\hline Reabilitação & 2,3 & $-0,25$ & $-0,66$ & 0,15 & 1,50 & 1 & 0,22 \\
\hline Farmácia & 3,7 & 0,56 & 0,34 & 0,78 & 24,95 & 1 & $<0,001$ \\
\hline Laboratório & 2,3 & $-0,13$ & $-0,37$ & 0,09 & 1,30 & 1 & 0,25 \\
\hline Radiologia & 4,7 & $-0,18$ & $-0,34$ & $-0,03$ & 5,51 & 1 & 0,01 \\
\hline Outra unidade & 21,5 & 0 & & & & & \\
\hline \multicolumn{8}{|l|}{ Cargo } \\
\hline Enfermeiro & 18,2 & $-0,06$ & $-0,19$ & 0,07 & 0,81 & 1 & 0,36 \\
\hline Técnico de Enfermagem & 31,1 & $-0,06$ & $-0,18$ & 0,05 & 1,10 & 1 & 0,29 \\
\hline Auxiliar de Enfermagem & 1,4 & $-0,60$ & $-1,32$ & 0,11 & 2,73 & 1 & 0,09 \\
\hline Médico do corpo clínico & 10,5 & $-0,21$ & $-0,37$ & $-0,04$ & 6,14 & 1 & 0,01 \\
\hline Médico residente & 0,5 & 0,54 & 0,25 & 0,83 & 13,87 & 1 & $<0,001$ \\
\hline Farmacêutico & 3,3 & $-0,67$ & $-0,84$ & $-0,49$ & 56,74 & 1 & $<0,001$ \\
\hline Nutricionista & 1,9 & $-0,12$ & $-0,53$ & 0,28 & 0,34 & 1 & 0,55 \\
\hline Assistente Social & 1,4 & $-1,16$ & $-1,50$ & $-0,83$ & 47,44 & 1 & $<0,001$ \\
\hline Odontólogo & 1 & 0 & & & & & \\
\hline Psicólogo & 1,4 & $-0,09$ & $-0,37$ & 0,18 & 0,46 & 1 & 0,49 \\
\hline Fisioterapeuta/Fonoaudiólogo/TO & 14 & 0,26 & 0,09 & 0,43 & 9,57 & 1 & $<0,01$ \\
\hline Técnico & 4,8 & 0,06 & $-0,08$ & 0,21 & 0,71 & 1 & 0,39 \\
\hline Administração & 1,9 & $-0,03$ & $-0,27$ & 0,20 & 0,07 & 1 & 0,78 \\
\hline Outros & 15,8 & 0 & & & & & \\
\hline Tempo de trabalho* & 12,81 & $-0,01$ & $-0,01$ & 0,01 & 0,93 & 1 & 0,33 \\
\hline Tempo na área* & 6,81 & 0,01 & $-0,006$ & 0,01 & 0,57 & 1 & 0,44 \\
\hline Horas de trabalho semanais ${ }^{*}$ & 35,85 & 0,01 & $-0,003$ & 0,01 & 0,53 & 1 & 0,46 \\
\hline Notificação de EA* & 8,25 & 0,001 & 0,001 & 0,01 & 65,55 & 1 & $<0,001$ \\
\hline
\end{tabular}

Intercepto $=$ ponto no modelo onde os valores das variáveis independentes equivalem a zero. $\mathrm{B}=$ Coeficiente de inclinação da variável nota geral de segurança do paciente em função da variável independente. ${ }^{*}=$ frequência média.

corpo clínico e gerencial quanto ao registro de incidentes e seus riscos nos serviços de saúde. Para El-Jardali et al. ${ }^{25}$, que avaliaram um hospital universitário na Arábia Saudita, percebe-se que as dimensões da CSP com melhores oportunidades de melhoria são a "resposta não punitiva a erros" "abertura para comunicações" e "dimensionamento de pessoal".
Nie et al. ${ }^{26}$ mostraram que existe diferenças na percepção da segurança de acordo com as unidades do serviço hospitalar, cargo e qualificação dos funcionários, ao estudarem 32 hospitais da China. Evidenciou-se que "respostas não punitivas" e "dimensionamento de pessoal" foram classificadas como fortalezas, além do "trabalho em equipe dentro/entre das unidades", "aprendizado 
organizacional/melhoria continuada" e "comunicação aberta". Em levantamento realizado com 45 hospitais na Holanda, 622 nos Estados Unidos e 74 em Taiwan revela-se que a principal dimensão carente de melhoria é de problemas em mudanças de turno e transições entre unidades/serviços, assim como relatam que existe grandes diferenças nas outras dimensões entre os países estudados ${ }^{27}$.

Quando foi analisado cada hospital separadamente, percebeu-se que apenas o hospital privado conseguiu atingir níveis de fortaleza (>75\%) na análise de respostas positivas (Tabela 3). Neste hospital, especificamente, as dimensões classificadas como fortalezas podem ser utilizadas como apoio para o processo de melhoria da segurança do paciente, visto que ela exerce uma força positiva na percepção de segurança dos profissionais como um todo ${ }^{28}$. O hospital estadual foi o único a apresentar todas as dimensões com percentual de respostas positivas compatível com a definição de fragilidade segundo a AHRQ $(<50 \%)$.

Seguindo a distribuição de respostas positivas, o hospital de gestão privada foi aquele que apresentou os melhores resultados. Este fato pode ser justificado devido a melhor estrutura existente. Esta instituição possui selo de acreditação em nível 1, apresentando, provavelmente, melhor estrutura para a segurança do paciente. Outro fator que pode estar associado a um melhor percentual de respostas positivas neste hospital é um eventual viés de informação relacionado com o vínculo empregatício, pois a instabilidade dos profissionais da instituição privada poderia inibir a exposição franca dos pontos negativos do hospital, apesar de que o termo de consentimento deixasse claro o anonimato dos respondentes e a confidencialidade das respostas. Esta hipótese pode ser testada em futuros estudos.

O hospital estadual é referência no atendimento ao trauma no Rio Grande do Norte e tem evidente sobrecarga de trabalho devido a insuficiência de capacidade estrutural do serviço para atender toda a demanda do estado. Há um histórico reconhecido de queixas dos próprios gestores a respeito de financiamento insuficiente para aquisição de insumos, medicamentos e equipamentos, além de dimensionamento de profissionais insuficiente para atender a demanda de trabalho. Em relação ao dimensionamento, esta é uma fragilidade da CSP percebida pelos profissionais $(30,1 \%$ de respostas positivas). Esses fatores podem explicar parte da discrepância de resultados dos hospitais federal e privado em relação ao hospital estadual (Tabela 3). Por exemplo, o hospital público federal é universitário e certificado como hospital de ensino, o que agrega mais recursos, pois recebe financiamento adicional de outros ministérios (ministério de educação, etc.), o que lhe diferencia do hospital estadual em questão ${ }^{29}$.

Dentro de cada serviço hospitalar, a CSP obteve diferentes avaliações de acordo com fatores associados, destacando-se a unidade de trabalho. Por exemplo, a farmácia foi a única avaliada positivamente (Tabela 4), corroborando com o estudo de Gama et al. ${ }^{30}$. Provavelmente, esse resultado deve-se ao uso de protocolos no controle de dispensação de medicamentos e materiais e ao menor contato dos funcionários com o paciente. Estas características podem ser opostas em outras unidades, tais como a de emergências, que teve a avaliação da CSP com menor percentual de respostas positivas (Tabela 4).

A identificação de fragilidades na CSP é uma oportunidade para melhoria da qualidade que deve ser aproveitada pelos NSP locais. O efeito positivo de intervenções de melhoria foi estudado por Burström et al. ${ }^{27}$, que implementaram um projeto de melhoria da qualidade em unidades de emergência de dois hospitais, um municipal e outro universitário, objetivando melhorar a CSP das equipes. O acompanhamento revelou evolução do trabalho em equipe na unidade, maior abertura para comunicação e melhor trabalho em equipe entre unidades do hospital universitário.

Apesar das contribuições, reconhecem-se algumas limitações no presente estudo. A primeira delas é o tamanho da amostra, a qual foi inferior em relação aos vários estudos feitos em outros países como na Espanha, onde participaram 1113 profissionais em oito hospitais públicos do país ${ }^{30}$. No entanto, os 215 funcionários respondentes entre os três estabelecimentos participantes atenderam às expectativas de comparar gestões hospitalares diferentes de acordo com os resultados obtidos. Em relação à extrapolação dos resultados a hospitais do mesmo tipo de gestão, é importante ter precaução, pois o estudo descreve de forma representativa e generalizável a realidade dos hospitais participantes nesta pesquisa e no contexto descrito, porém as características podem variar em outras regiões do país, cidades ou instituições do mesmo tipo de gestão.

Outra limitação é o baixo percentual de respostas dos profissionais, mesmo após o reenvio do questionário para os mesmos endereços eletrônicos em três tentativas e ida presencial aos hospitais. Assim como em todos os inquéritos voluntários, as estimativas podem estar superestimadas, pois as respostas podem ter sido dadas por aquelas pessoas mais comprometidas com o 
hospital e a segurança do paciente. Neste contexto o National Quality Forum dos Estados Unidos inclusive considera a porcentagem de resposta como um indicador do nível de cultura de segurança, pois quanto maior a cultura, mais os profissionais se sentem motivados a responder este tipo de questionário ${ }^{18}$. $\mathrm{O}$ fato do software utilizado não distinguir quais foram os retornos por email, ou com preenchimento presencial no hospital, é outro fator limitante ligado ao baixo percentual de resposta.

A responsividade à avaliação da CSP é variável no mundo. Fujita et al..$^{31}$ revelaram que nos Estados Unidos a taxa de resposta é em torno de $35,2 \%$, no Japão, de $66,5 \%$ e em Taiwan, de $85,7 \%$. Klopper-Kes et al..$^{32}$ obtiveram taxa de resposta de $27 \%$ na Alemanha, já na China ${ }^{26}$ foi de $77 \%$. Parece que o clima organizacional para a segurança do paciente em serviços do Brasil está aquém do apresentado internacionalmente (percentual de resposta ao questionário 13,6\%), sendo necessárias medidas urgentes da liderança para promover maior envolvimento dos profissionais e alcance de um percentual de resposta adequado ${ }^{33}$.

É necessária a realização de novas pesquisas que englobem mais hospitais de outras regiões do país, para melhorar a representatividade quantitativa e qualitativa dos achados; disponibilizar ferramentas para o monitoramento da CSP de modo rápido e contínuo, com auxílio de tecnologias validadas para este fim; aprofundar o estudo dos fatores contextuais de cada tipo de gestão, além dos abordados neste estudo, que influenciam os diferentes níveis de CSP; e, por fim, em relação a políticas públicas, precisa-se regular este tipo de avaliação nos hospitais brasileiros, juntamente com a divulgação das informações produzidas, para orientação e evolução do recente Programa Nacional de Segurança do Paciente.

\section{Conclusão}

As crenças, atitudes e percepções dos profissionais de saúde em relação a segurança do paciente ainda são incipientes nos hospitais avaliados. Embora a maioria dos profissionais classifique a segurança como boa, as fortalezas nas dimensões da cultura de segurança foram escassas e concentradas em um hospital privado.

O estudo permite a identificação de fragilidades que devem receber planejamento de intervenções de melhoria e lança hipóteses que devem ser confirmadas em estudos futuros. As intervenções devem considerar o tipo de gestão do serviço hospitalar, a profissão, cargo e a quantidade de notificação de incidentes, pois parecem interferir na percepção geral dos profissionais. $\mathrm{O}$ nível de CSP foi crescente nos hospitais de gestão estadual, federal e privado.

\section{Colaboradores}

LEL Andrade participou da análise e interpretação dos dados, redação do artigo e revisão crítica e aprovação da versão a ser publicada. JM Lopes participou da análise e interpretação dos dados, revisão crítica, e aprovação da versão a ser publicada. MCM Souza Filho, RF Vieira Júnior, LPC Farias e CCM Santos participaram do delineamento, redação do artigo e aprovação da versão a ser publicada. ZAS Gama participou da concepção, revisão crítica, e aprovação da versão a ser publicada.

\section{Referências}

1. Kohn LT, Corrigan JM, Donaldson MS, editors. To err is human: building a safer health system. Washington: National Academy Press, Institute of Medicine; 1999.

2. Mendes W, Martins M, Rozenfeld S, Travassos C. The assessment of adverse events in hospitals in Brazil. Int Qual in Health Care 2009; 21(4):279-284.

3. World Health Organization (WHO). The Conceptual Framework for the International Classification for $\mathrm{Pa}$ tient Safety v1.1. Final Technical Report and Technical Annexes. Geneva: WHO; 2009.

4. Choudhry RM, Fang D, Mohamed S. The nature of safety culture: A survey of the state-ofthe-art. Saf Sci 2007; 45(10):993-1012.

5. Runciman W, Hibbert P, Thomson R, Van Der Schaaf T, Sherman H, Lewalle P. Towards an International Classification for Patient Safety: key concepts and terms. Int J Qual Health Care 2009; 21(1):18-26. 
6. International Atomic Energy Agency (INSAG). Safety Culture: A report by the International Nuclear Safety Advisory Group. Viena: INSAG; 1991. Safety Series No. 75-INSAG-4.

7. Nieva VF, Sorra J. Safety culture assessment: A tool for improving patient safety in healthcare organizations. Qual Saf Health Care 2003; 12(Supl. 2):ii17-23.

8. Agência Nacional de Vigilância Sanitária (Anvisa). Implantação do Núcleo de Segurança do Paciente em Serviços de Saúde. Série Segurança do Paciente e Qualidade em Serviços de Saúde. Brasília: Anvisa; 2014.

9. Guldenmund FW. The use of questionnaires in safety culture research - an evaluation. Saf Sci 2007; 45(6): 723-743.

10. Singla AK, Kitch BT, Weissman JS, Campbell EG. Assessing patient safety culture: A review and synthesis of the measurement tools. J Patient Saf 2006; 2(3):105115 .

11. Hellings J, Schrooten W, Klazinga N, Vleugels A. Challenging patient safety culture: survey results. Int $J$ Health Care Qual Assur 2007; 20(7):620- 632.

12. Tomazoni A, Rocha PK, Souza S, Anders JC, Malfussi HFC. Cultura de segurança do paciente em unidades de terapia intensiva neonatal: perspectivas da equipe de enfermagem e médica. Rev LatinoAm Enferm 2014; 22(5):755-763.

13. Tomazoni A, Rocha PK, Kusahara DM, Souza AIJ, Macedo TR. Avaliação da cultura de segurança do paciente em terapia intensiva neonatal. Texto \& Contexto Enferm. 2015; 24(1):161-9.

14. Marinho MM, Radünz V, Barbosa SFF. Avaliação da cultura de segurança pelas equipes de enfermagem de unidades cirúrgicas. Texto Contexto Enferm 2014; 23(3):581-590.

15. Da Correggio TC, Amante LN, Barbosa SFF. Avaliação da cultura de segurança do paciente em Centro Cirúrgico. Rev SOBECC 2014; 19(2):67-73.

16. Brasil. Portaria No 529, de $1^{\circ}$ de abril de 2013. Institui o Programa Nacional de Segurança do Paciente (PNSP). Diário Oficial da União 2013; 2 abr.

17. Agência Nacional de Vigilância Sanitária. Resolução RDC No 36, de 25 de julho de 2013. Institui ações para a segurança do paciente em serviços de saúde e dá outras providências. Diário Oficial da União 2013; 26 jul.

18. National Quality Forum (NQF). Safe Practices for Better Healthcare-2010 Update: A Consensus Report. Washington: NQF; 2010.

19. Reis CT. A cultura de segurança do paciente: validação de um instrumento de mensuração para o contexto hospitalar brasileiro [tese]. Rio de Janeiro: Escola Nacional de Saúde Pública Sergio Arouca; 2013.

20. Gama ZAS, Batista AM, Silva IG, Souza RM, Freitas MR. Adaptação transcultural da versão brasileira do Hospital Survey on Patient Safety Culture: oportunidades de melhoria. Cad Saude Publica 2013; 29(7):14731477.

21. Sorra J, Nieva V, Famolaro T, Dyer N. Hospital survey on patient safety culture 2007 comparative database report. Rockville: Agency for Healthcare Research and Quality; 2007. (AHRQ Publication, 08-0039).
22. Sorra J, Famolaro T, Dyer N, Nelson D, Smith SA. Hospital survey on patient safety culture 2012 user comparative database report. Rockville: Agency for Healthcare Research and Quality; 2012. (AHRQ Publication, 120017).

23. Sorra JS, Nieva VF. Hospital survey on patient safety culture. Rockville: Agency for Health Care Research and Quality; 2004. (AHRQ Publication, 04-0041).

24. Wiegmann DA, Zhang H, von Thaden T, Sharma G, Mitchell A. A synthesis of safety culture and safety climate research. Savoy: University of Illinois at Urbana-Champaign; 2002. (Technical Report ARL- 02-3/ FAA-02-2).

25. El-Jardali F, Sheikh F, Garcia NA, Jamal D, Abdo A. Patient safety culture in a large teaching hospital in Riyadh: baseline assessment, comparative analysis and opportunities for improvement. BMC Health Serv Res 2014; 14:122.

26. Nie Y, Mao Y, Cui H, He S, Li J, Zhang M. Hospital survey on patient safety culture in China. BMC Health Serv Res 2013; 13:228.

27. Burström L, Letterstål A, Engström ML, Berglund A, Enlund M. The patient safety culture as perceived by staff at two different emergency departments before and after introducing a flow-oriented working model with team triage and lean principles: a repeated cross-sectional study. BMC Health Serv Res 2014; 14:296.

28. Wagner C, Smits M, Sorra J, Huang CC. Assessing patient safety culture in hospitals across countries. Int $\mathrm{J}$ Qual Health Care 2013; 25(3):213-221.

29. La Forgia GM, Couttolenc BF. Desempenho hospitalar no Brasil: em busca da excelência. In: La Forgia GM, Couttolenc BF. O setor hospitalar brasileiro: estrutura, financiamento, gasto e resultados. São Paulo: Singular; 2009. p. 28-33.

30. Gama ZAS, Oliveira ACS, Hernández PJS. Cultura de seguridade del paciente y factores asociados en una red de hospitales públicos españoles. Cad Saude Publica 2013; 29(2):283-293.

31. Fujita S, Seto K, Ito S, Wu Y, Chiu-Chin Huang, Hasegawa $\mathrm{T}$. The characteristics of patient safety culture in Japan, Taiwan and the United States. BMC Health Serv Res 2013; 13:20

32. Klopper-Kes HAHJ, Siesling S, Meerdink N, Wilderom CPM, van Harten HV. Quantifying culture gaps between physicians and managers in Dutch hospitals: a survey. BMC Health Serv Res 2010; 10:86.

33. Robb G, Seddon M. Measuring the safety culture in a hospital setting: a concept whose time has come? N Z Med J 2010; 123(1313):66-76.

Artigo apresentado em 11/08/2015

Aprovado em 20/01/2016

Versão final apresentada em 22/01/2016 